\title{
Estimation of a Cable Resistance Profile with Readaptation of Mismatched Measurement Instrument
}

\author{
N. Berrabah *†
}

\author{
Q. Zhang ${ }^{\dagger}$
}

\begin{abstract}
As the cumulative length of electric cables in modern systems is growing and as these systems age, it becomes of crucial importance to develop efficient tools to monitor the condition of wired connections. Therein, in contrast to hard faults (open or short circuits), the diagnosis of soft-faults requires a particular effort. Indeed, these faults are more difficult to detect, yet they are sometimes early warning signs of more important failures. In a previous paper, we proposed a method to compute the resistance profile $R(z)$ of a cable from reflectometry measurements made at both ends of the cable. It enables detection, localization and estimation of dissipative soft-faults. In this paper, we propose an improvement of this method in case of impedance mismatch between the measurement instrument and the cable, based on a pre-processing of the measurement data before running the estimation computations. It aims at reducing the impedance mismatch between instrumentation and the cable under test without physical intervention on the test fixtures. In addition, this paper proposes a measurement procedure allowing us to get the two-ends reflectometry measurements without actually connecting both ends of the cable under test to a single instrument.
\end{abstract}

\section{INTRODUCTION}

In complex systems involving a large number of wired connections, monitoring cable failures is becoming an important issue in order to ensure safe operations, high performance and cost effectiveness. To this end, it is useful to develop monitoring and diagnosis tools that enable to detect, localize and characterize faults at the earliest possible stage [1]. Cable faults due to aging and harsh operating environment consist materially in a modification of the original physical properties of the cable. These changes may be localized on a point or short section of a line or distributed on a longer length. They can either lead to interruption of power or information transmission, in which case we speak of hard fault, or only consist in slight modifications of the cable properties, without significant impacts on the whole system operation, and give rise to soft faults. Hard faults are well handled by existing methods among which reflectometry [2] is one of the most used. However, soft faults re-

${ }^{*}$ EDF R\&D, France, e-mail: nassif.berrabah@edf.fr, maud.franchet@edf.fr, denis.vautrin@edf.fr.

${ }^{\dagger}$ I4S, Institut National de Recherche en Informatique et Automatique, France, e-mail: qinghua.zhang@inria.fr, nassif . berrabah@inria.fr.

\author{
M. Franchet * D. Vautrin *
}

main hard to detect with the most classical techniques. In the past few years several attempts were made to improve reflectometry measurements analysis, mainly using signal processing tools. In spite of these efforts, reflectometry still fails at detecting some of the softest faults [3]. Among soft-faults, resistive ones are related to overheating that reduces the current-carrying capacity of the cable and also threatens its isolation integrity. In a previous paper [4] we proposed a method to estimate the per unit length resistance profile $R(z)$ of a cable from reflectometry measurements made at both ends of the cable. This constitutes a mean of diagnosis of resistive faults either localized or distributed. Nevertheless, its accuracy is sensitive to mismatch between the cable under test and the measurement instrument, typically a Vector Network Analyzer (VNA). In this paper, we present a solution to this drawback based on a pre-processing of the measured data before application of the estimation procedure. In addition, we show how the two-ends measurements required by the estimation method can be obtained by connecting a single end of the cable under test to a VNA and by switching the other end between three different loads.

The paper is organized as follows. The second section recalls the main elements of our resistance profile estimation method. In section 3 , we explain why impedance discontinuity deteriorates the estimation accuracy and we present the solution that we designed. Section 4 details a measurement procedure that saves the operator from having to physically connect the VNA to both ends. Results are presented in section 5. Finally, a conclusion is drawn.

\section{Resistance Profile Estimation Method}

Inverse Scattering for Transmission Lines (ISTL) is an algorithm initially designed to estimate the spatially distributed characteristic impedance of a transmission line assumed lossless [5]. More recently, it has been used as part of an advanced method for the estimation of ohmic losses in lossy lines based on reflectometry measurements at both ends of the lines under test [4]. In this section we briefly recall how this estimation is obtained. 


\subsection{RLCG Model}

The estimation of the per unit length resistance $R(z)$ of a cable consists in the resolution of an inverse problem. The associated forward model is widely known as the RLCG model, or telegrapher's equations. It is described by equations (1):

$$
\begin{aligned}
& \frac{\partial u}{\partial z}(z, t)=-R(z) i(z, t)-L(z) \frac{\partial i}{\partial t}(z, t) \\
& \frac{\partial i}{\partial z}(z, t)=-G(z) u(z, t)-C(z) \frac{\partial u}{\partial t}(z, t)
\end{aligned}
$$

In this representation, $u(z, t)$ (respectively $i(z, t)$ ) denotes the voltage (respectively current) at position $z$ and time $t$. A transmission line is fully characterized by the distributed $R(z), L(z), C(z)$ and $G(z)$ parameters which represent the resistance per unit length of conductors, the inductance per unit length, the capacitance per unit length, and the conductance per unit length of insulation respectively. The approach adopted in [4] consists in obtaining an estimation of the parameter $R(z)$ from S-parameters (scattering parameters) measured on the cable.

\subsection{Estimation of $R(z)$}

The estimation of the resistance profile is based on the two reflection coefficients measured at the two ends of a cable. The coefficient from one end (let us call it the left end) is obtained with a VNA connected to the said end while a matched load is connected to the other end. The second coefficient (from the right end) shall be obtained by interchanging the roles of each end. In practice these coefficients are typically measured in a single experiment with a VNA connected at both ends. Then the resistance profile is computed following the three-steps procedure below:

1. From the measured reflection coefficients, we calculate two estimations $Z_{0}^{(l)}(z)$ and $Z_{0}^{(r)}(z)$ of its characteristic impedance, using ISTL as if the cable was lossless (if it actually was, the two results would be identical).

2 . Under the low-reflection (soft-fault only) assumption, $Z_{0}^{(l)}(z)$ and $Z_{0}^{(r)}(z)$ are approximately equal to the apparent impedances $Z^{(l)}(z)$ and $Z^{(r)}(z)$ defined as the ratio of voltage over current at position $z$ in each of the measurement experiment:

$$
Z_{0}^{(l)}(z) \approx Z^{(l)}(z) \quad \text { and } \quad Z_{0}^{(r)}(z) \approx Z^{(r)}(z)
$$

Note that in case of hard fault, this assumption is not valid but classical methods such as TDR are suitable to detect it.
3. The estimation of $R(z)$ is finally given by:

$R(z) \approx \frac{\left[Z^{(l)}(z)-Z^{(r)}(z)\right]-\left[Z^{(l)}(z+\delta z)-Z^{(r)}(z+\delta z)\right]}{2 \delta z}$

where $\delta z$ is the discretization step.

This method is computationally fast and produces accurate results when measurements are made with an instrument matched to the impedance of the tested line. In order to limit as much as possible the effect of impedance mismatch that may exist between instrumentation and the transmission line, section 3 proposes a data preprocessing procedure.

\section{Readaptation of Impedance Mismatches}

As most standard VNAs have a reference impedance of 50 ohms, whereas cables of different impedances are used, one may have to deal with an impedance mismatch at the connection point. In this section we will explain how this can affect the final estimation of $R(z)$ and propose a solution based on the pre-processing of measured data.

\subsection{Effects of Impedance Mismatch}

One immediate trouble caused by an impedance mismatch at the instrument port is the degradation of the Signal to Noise Ratio. Indeed this impedance discontinuity creates reflections, and as a consequence reduces the amount of energy sent into the cable to perform the measurement. The second issue in our case is inherent to the method itself. Indeed, the theory behind the ISTL algorithm supposes that the impedance varies continuously along the cable. In practice the algorithm is robust and tolerates some impedance discontinuity. Yet, it produces an error in the impedance estimation. The more severe the impedance discontinuity, the more important the error is. In what follows, we propose a data pre-processing method to re-adapt the Sparameters measurements in order to compensate any impedance discontinuity.

\subsection{Re-adaptation method}

The initial S-parameters are measured with respect to the impedance of the VNA ports denoted here with $Z_{01}$ (port connected to the left end) and $Z_{02}$ (right end). The proposed pre-processing aims at transforming them to a new set of S-parameters, as if it was measured with a matched instrument. The proposed method relies on the conversion formulas [6] between different equivalent characterizations of two-port networks, like S-parameters, ABCD-parameters (chain, cascade or transmission 
parameters), Z-parameters (impedance) and Yparameters (admittance). Some of these parameters are functions of a reference impedance, as in the case of S-parameters, while others are not. The main idea here, is to convert the measured matrix of S-parameters to the equivalent Z-parameters, which are independent of any reference impedance, and then to convert them back to another matrix of S-parameters with respect to a desired reference impedance. S-parameters are converted to Zparameters as follows:

$$
\begin{aligned}
Z_{11} & =\frac{\left(Z_{01}^{*}+S_{11} Z_{01}\right)\left(1-S_{22}\right)+S_{12} S_{21} Z_{01}}{\left(1-S_{11}\right)\left(1-S_{22}\right)-S_{12} S_{21}} \\
Z_{12} & =\frac{2 S_{12} \sqrt{\Re\left(Z_{01}\right) \Re\left(Z_{02}\right)}}{\left(1-S_{11}\right)\left(1-S_{22}\right)-S_{12} S_{21}} \\
Z_{21} & =\frac{2 S_{21} \sqrt{\Re\left(Z_{01}\right) \Re\left(Z_{02}\right)}}{\left(1-S_{11}\right)\left(1-S_{22}\right)-S_{12} S_{21}} \\
Z_{22}= & \frac{\left(1-S_{11}\right)\left(Z_{02}^{*}+S_{22} Z_{02}\right)+S_{12} S_{21} Z_{02}}{\left(1-S_{11}\right)\left(1-S_{22}\right)-S_{12} S_{21}}
\end{aligned}
$$

and the reverse conversion is based on:

$$
\begin{aligned}
S_{11} & =\frac{\left(Z_{11}-Z_{c 1}^{*}\right)\left(Z_{22}+Z_{c 2}\right)-Z_{12} Z_{21}}{\left(Z_{11}+Z_{c 1}\right)\left(Z_{22}+Z_{c 2}\right)-Z_{12} Z_{21}} \\
S_{12} & =\frac{2 Z_{12} \sqrt{\Re\left(Z_{c 1}\right) \Re\left(Z_{c 2}\right)}}{\left(Z_{11}+Z_{c 1}\right)\left(Z_{22}+Z_{c 2}\right)-Z_{12} Z_{21}} \\
S_{21} & =\frac{2 Z_{21} \sqrt{\Re\left(Z_{c 1}\right) \Re\left(Z_{c 2}\right)}}{\left(Z_{11}+Z_{c 1}\right)\left(Z_{22}+Z_{c 2}\right)-Z_{12} Z_{21}} \\
S_{22}= & \frac{\left(Z_{11}+Z_{c 1}\right)\left(Z_{22}-Z_{c 2}^{*}\right)-Z_{12} Z_{21}}{\left(Z_{11}+Z_{c 1}\right)\left(Z_{22}+Z_{c 2}\right)-Z_{12} Z_{21}}
\end{aligned}
$$

The new S-parameters should be expressed with respect to the characteristic impedance of the transmission line under test at each connection point $Z_{c 1}=Z_{c}(z=0)$ and $Z_{c 2}=Z_{c}\left(z=z_{L}\right)$, which are supposed known a priori.

\section{S-Parameters From Single End Measure- ment and Load Variation}

In applications to electrical cable fault diagnosis, S-parameters are typically measured by connecting simultaneously two ports of a VNA to the two ends of a cable. However, this cannot always be done, especially when dealing with long lines that cannot have their two ends connected to the same instrument at the same time. In this section, an alternative method is proposed by connecting a single VNA port to one end of a cable while switching the other end between three different loads. At every tested frequency, the parameters $S_{11}, S_{22}$ and the product $S_{12} S_{21}$ are derived from three single port measurements, each corresponding to a different load.
With a single port connected to one end of the cable under test, the VNA provides a measurement of the reflection coefficient $\Gamma_{1}^{\left(\Gamma_{L}\right)}$ which is related to the S-parameters of the cable through (6):

$$
\Gamma_{1}^{\left(\Gamma_{L}\right)}=S_{11}+\frac{S_{21} S_{12} \Gamma_{L}}{1-S_{22} \Gamma_{L}}
$$

where $\Gamma_{L}$ represents the reflection coefficient at the load abscissa $z_{L}$. It is defined for a load impedance $Z_{L}$ as:

$$
\Gamma_{L}=\frac{Z_{L}-Z_{0}\left(z_{L}\right)}{Z_{L}+Z_{0}\left(z_{L}\right)}
$$

Note that (6) is only equal to $S_{11}$ if $\Gamma_{L}=0$, that is to say, if the load is matched to the cable. Otherwise, the measurement gives an equation that relates linearly together $S_{11}, S_{22}$, and the product $S_{12} S_{21}$. The equations corresponding to three different values of $\Gamma_{L}$, are linearly independent so that the three unknowns $S_{11}, S_{22}$ and $S_{12} S_{21}$ can be solved.

A full matrix of S-parameters cannot be obtained in this fashion, but the three numbers that we get are sufficient to estimate $R(z)$, including if necessary the readaptation process presented in section3. Indeed, in (4) replace $(4 \mathrm{~b})$ and $(4 \mathrm{c})$ by their product, then one can calculate $Z_{11}, Z_{22}$, and $Z_{12} Z_{21}$ from the data available. Next, from this intermediate step, and thanks to the multiplication of $(5 \mathrm{~b})$ with (5c), the readapted parameters $S_{11}, S_{22}$, and $S_{12} S_{21}$ can be computed. In the next session we demonstrate the efficiency of this method through numerical results.

\section{$5 \quad$ Results}

In this section, numerical results are provided to demonstrate the efficiency of the proposed procedure and its added value to the diagnosis of ohmic losses in electrical cables. Results are also presented to confirm the feasability of the proposed single-end measurement procedure.

We have simulated the measurements made on a $10 \mathrm{~m}$-long cable of characteristic impedance $105 \Omega$, with several resistive faults of different profiles. For each case the measurements were simulated with two methods: one corresponding to a two-ports VNA of reference impedance $50 \Omega$ connected at both ends of the cable, and another corresponding to the procedure explained in section 4 . In the second method, the three loads were taken to be $50 \Omega$, a short circuit and an open circuit which correspond to the available standards in most calibration kits. Then we estimated the resistance profile according to $(3)$ :

(a) Directly from the simulated data. 


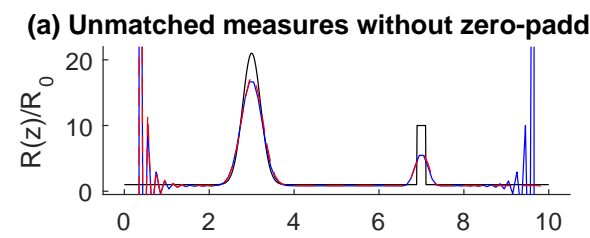

(b) Unmatched measures with zero-padding

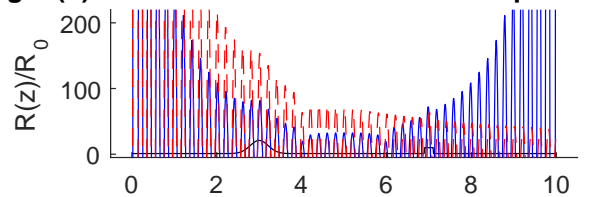

(c) Re-adapted measures without zero-padding
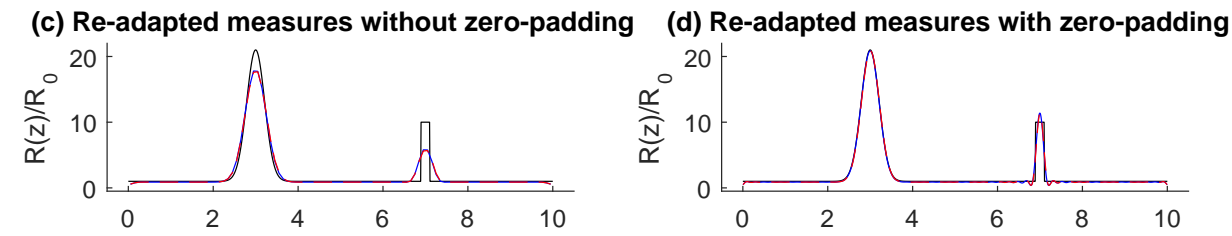

Figure 1: Effect of the re-adaptation procedure on the estimation of the resistance per unit length $R(z)$

(b) From the simulated data zero-padded to increase the resolution of the estimated ohmic losses.

(c) From the simulated data re-adapted following the procedure proposed in this paper.

(d) From the simulated data re-adapted and zeropadded.

The results obtained are visible on figure 1 . The ohmic losses are characterized by the normalized per unit length resistance $\frac{R(z)}{R_{0}}$. Where $R_{0}$ is the nominal per unit length resistance of the simulated cable and $z$ represents the position on the cable. The black curves represent the simulated profile $R(z) / R_{0}$, whereas the blue and dashed red curves present the estimated profiles $R(z) / R_{0}$ obtained with the two-ports VNA measurements and the single port method respectively. The results of the upper row are obtained from the unmatched measurements, and those of the lower row from readapted measurements.

The improvements brought by impedance readaption are clear. Indeed, comparing graphs (a) and (c), we can readily see how the results are enhanced when applying the prepocessing procedure. Moreover, the comparison between (b) and (d) illustrates how it prevents the occurence of ringing artifacts when the measured data is zero-padded in order to increase the resolution of the estimated ohmic losses (right side plots).

Moreover, these simulations illustrate the performance of the procedure detailed in section 4 as in each case, the red and blue curves are almost identical.

\section{Conclusion}

We have explained how a pre-processing procedure applied to the measurements can eliminate an impedance discontinuity at the VNA port, and therefore enhance the accuracy of the resistive losses estimation. This procedure also has the advantage of preventing numerical errors introduced by zero-padding in presence of discontinuities. $\mathrm{Nu}$ merical results were shown to support the theory. In a future work the problem of discontinuities introduced by connectors shall be addressed.

\section{References}

[1] C. Furse, "Down to the Wire," IEEE Spectrum, pp. 34-39, 2001.

[2] F. Auzanneau, "Wire Troubleshooting and Diagnosis - Review and Perspectives," Progress In Electromagnetics Research, vol. 49, no. February, pp. 253-279, 2013.

[3] L. A. Griffiths, R. Parakh, C. Furse, and B. Baker, "The invisible fray: a critical analysis of the use of reflectometry for fray location." IEEE Sensors Journal, vol. 6, no. 3, pp. 697706, 2006.

[4] N. Berrabah, M. Franchet, D. Vautrin, and Q. Zhang, "Estimation of Distributed and Lumped Ohmic Losses in Electrical Cables," in IEEE Conference on Antenna Measurements and Applications, Syracuse, 2016.

[5] Q. Zhang, M. Sorine, and M. Admane, "Inverse scattering for soft fault diagnosis in electric transmission lines," IEEE Transactions on Antennas and Propagation, vol. 59, no. 1, pp. 141-148, 2011.

[6] D. A. Frickey, "Conversions Between S, Z, Y, h, ABCD, and T Parameters which are Valid for Complex Source and Load Impedances," IEEE Transactions on Microwave Theory and Techniques, vol. 42, no. 2, pp. 205-211, 1994. 